\title{
Magnetized QCD phase diagram: critical end points for the strange quark phase transition driven by external magnetic fields
}

\author{
Pedro Costa*, Márcio Ferreira and Constança Providência \\ CFisUC, Department of Physics, University of Coimbra, P-3004 - 516 Coimbra, Portugal \\ E-mail: pcostaduc.pt mferreiradteor.s.uc.pt, cpefis.uc.pt
}

In this work we examine possible effects of an external magnetic field in the strongly interacting matter phase diagram. The study is performed using the Polyakov-Nambu-Jona-Lasinio model. Possible consequences of the inverse magnetic catalysis effect on the phase diagram at both finite chemical potential and temperature are analyzed. We devote special emphasis on how the location of the multiple critical end points (CEPs) change in a magnetized medium: the presence of an external magnetic field induces several CEPs in the strange sector, which arise due to the multiple phase transitions that the strange quark undergoes. We also study the deconfinement transition which turns out to be less sensitive to the external magnetic field when compared to the quark phase transitions. The crossover nature of the deconfinement is preserved over the whole phase diagram.

XVII International Conference on Hadron Spectroscopy and Structure - Hadron2017

25-29 September, 2017

University of Salamanca, Salamanca, Spain

* Speaker. 
The QCD phase diagram and the respective chiral critical end point (CEP), belong to a set of quantum field theoretical phenomena that are affected by the presence of external magnetic fields

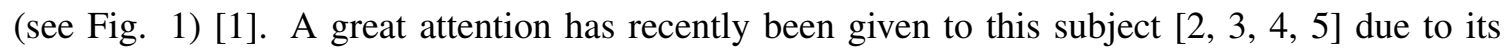
relevance for heavy ion collisions (HIC) measurements [ [G], for the physics of compact stars [ $[$ ], and for the understanding of the primordial stages of the universe [ []$]$. Having this in mind, different scenarios involving regions of the phase diagram in the presence of external magnetic fields were studied using the Polyakov-Nambu-Jona-Lasinio (PNJL) model with (2+1)-flavors [Q⿴囗⿰丿㇄口, 四, 四]. In some of these works, it was analyzed how the location of the CEP depends on the presence of magnetic fields. In $[Q]$, for example, it was shown that large isospin asymmetry moves the CEP to smaller temperatures leading, eventually, to its disappearance from the phase diagram. Nevertheless, a first-order phase transition will be restored in the phase diagram if a strong enough magnetic field is present.

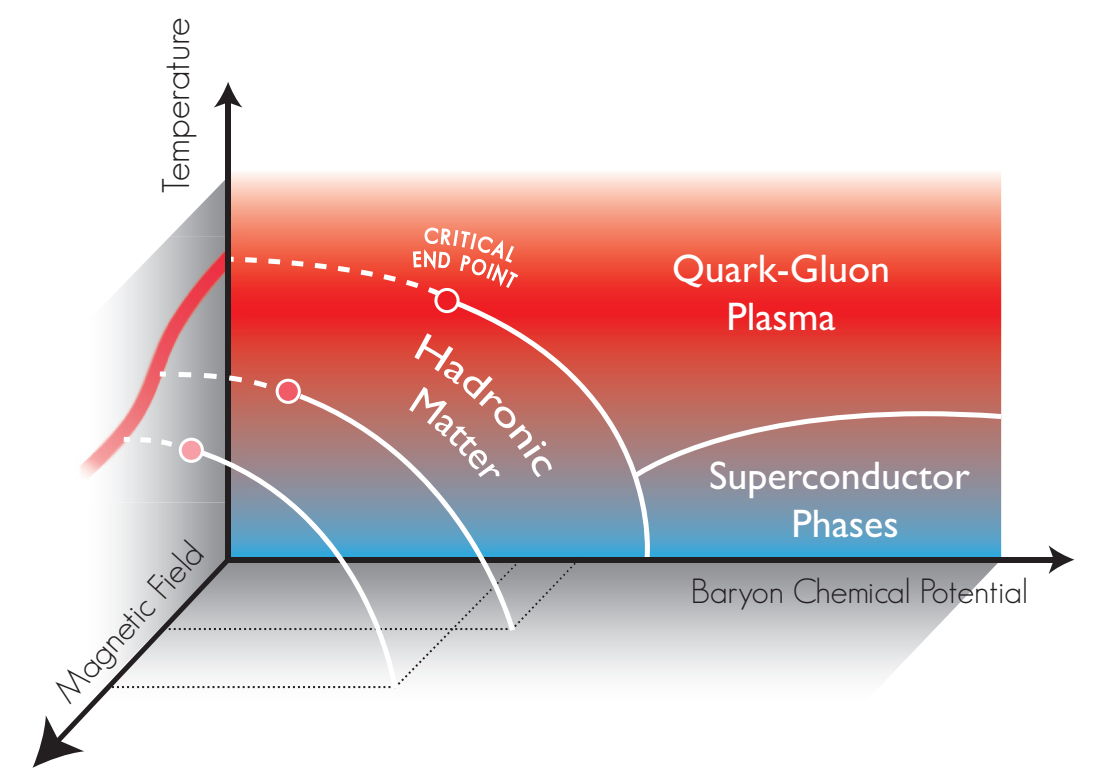

Figure 1: Schematic structure of the QCD matter in the presence of an external magnetic field.

One well known and understood mechanism induced by the presence of an external magnetic field is the catalyzing effect on the dynamical chiral symmetry breaking, the so-called Magnetic Catalysis (MC) effect [102]. Lattice QCD (LQCD) studies at finite temperature have shown, however, that the magnetic field has an interesting behavior in the transition temperature region: instead of catalyzing, it weakens the dynamical chiral symmetry breaking, the so-called Inverse Magnetic Catalysis (IMC) [[3]]. Several explanations have been proposed to clarify this unexpected effect [四]. Motivated by LQCD calculations reported in [14]], the IMC effect was incorporated successfully for the first time in [వ]]: with the introduction of an indirect weakening of the model scalar coupling, $G_{s}$, with $B$ (via the Polyakov potential), it was obtained an extended (2+1)-PNJL model that presented an IMC effect for the quarks condensates at finite temperature. Later in [B]], we considered the screening effects of strong interactions through the scalar coupling $\left(G_{s}(e B)\right)$, achieving a qualitative agreement with LQCD results.

At finite temperature and density/chemical potential, we now study how the IMC mechanism, 
via a magnetic field dependent coupling $G_{s}(e B)$, affects the first order region and the position of the CEP. In Fig. $\square$ we present the phase diagram $\left(T-\mu_{B}\right.$ plane - upper panels; $T-\rho_{B}$ plane lower panels) for three different cases: $e B=0$ - left panels; $e B=0.3 \mathrm{GeV}^{2}$ and $G_{s}^{0}=$ const. (no IMC effect) - middle panels; $e B=0.3 \mathrm{GeV}^{2}$ and $G_{s}(e B)$ (with IMC effect) - right panels. From the upper panels, we conclude that the presence of a magnetic field will: a) enlarge the spinodal region for the light sector, being more pronounced without an IMC mechanism (middle panel); $b$ ) move the light CEP to lower values of $\mu_{B}$, being stronger when the IMC effect is present (right panel); c) generate multiple first-order phase transitions for the strange sector with the respective appearance of multiple CEPs in this sector (for $e B \gtrsim 0.4 \mathrm{GeV}^{2}$, only one strange CEP exists). Instead of a single first-order phase transition connecting the vacuum phase to the chirally restored phase, several intermediate first-order phase transitions take place that are generated by Landau quantization, induced by the magnetic field presence, and a succession of partial restorations of the chiral symmetry.
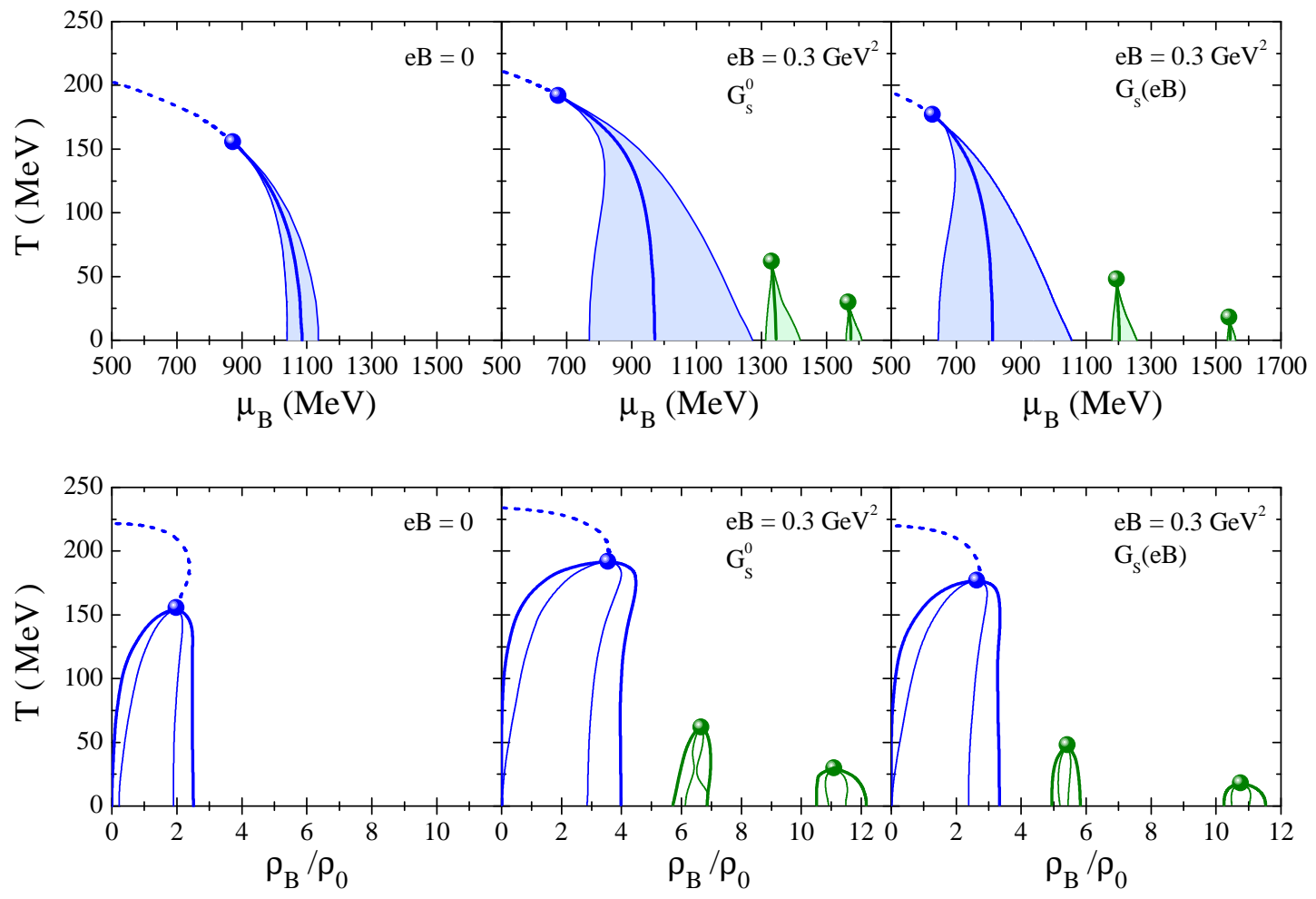

Figure 2: The $T-\mu_{B}$ (top panels) and $T-\rho_{B}$ (bottom panels) diagrams for: $e B=0$ (left); $e B=0.3 \mathrm{GeV}^{2}$ and $G_{s}^{0}=$ const., scenario with no IMC mechanism (middle); and $e B=0.3 \mathrm{GeV}^{2}$ and $G_{s}(e B$ ), scenario with IMC mechanism (right panel). The baryonic density $\rho_{B}$ is represented in units of saturation density, $\rho_{0}=0.16 \mathrm{fm}^{3}$.

Another relevant aspect for both, light and strange, transitions is that for stronger magnetic fields the spinodal region is enlarged, being this region bigger for $G_{s}=G_{s}^{0}$ [ए5]]. The first-order lines are moved to lower baryonic chemical potentials. From the bottom panels of Fig. Z, we also conclude that the upper baryonic densities at which the onset of both spinodal and binodal regions 
occur increase with $B$ for both cases. Moreover, the spinodal region for the strange quark is much smaller than for the light quarks and is located at higher values of $\rho_{B}$.

Concerning the CEPs, we present the results in Fig. B (left panel). We start by comparing the CEP's position for the light sector ( $u$ and $d$ quarks) with and without the IMC mechanism. For magnetic fields lower than $0.3 \mathrm{GeV}^{2}$, we have found that the presence of an IMC mechanism has a small effect in the CEP position, i.e., the CEPs move towards higher values of $T$ and $\mu_{B}$ in both scenarios (see red and black curves). For higher magnetic fields, however, the CEP is moved to lower $\mu_{B}$ with increasing magnetic fields for $G_{s}(e B)$, while the temperature remains almost unchanged [ए]]. Indeed, the $G_{s}(e B)$ results indicate that, for high enough magnetic fields, the CEP goes towards the $\mu_{B}=0$ axis, and the crossover transition in this axis will eventually turn into a first-order phase transition. This behavior is distinct from the one obtained when the IMC effect is absent $\left(G_{s}^{0}\right)$ : above a critical magnetic field strength, the CEP location is shifted to higher values of $T$ and $\mu_{B}$ with increasing magnetic field [Q] .
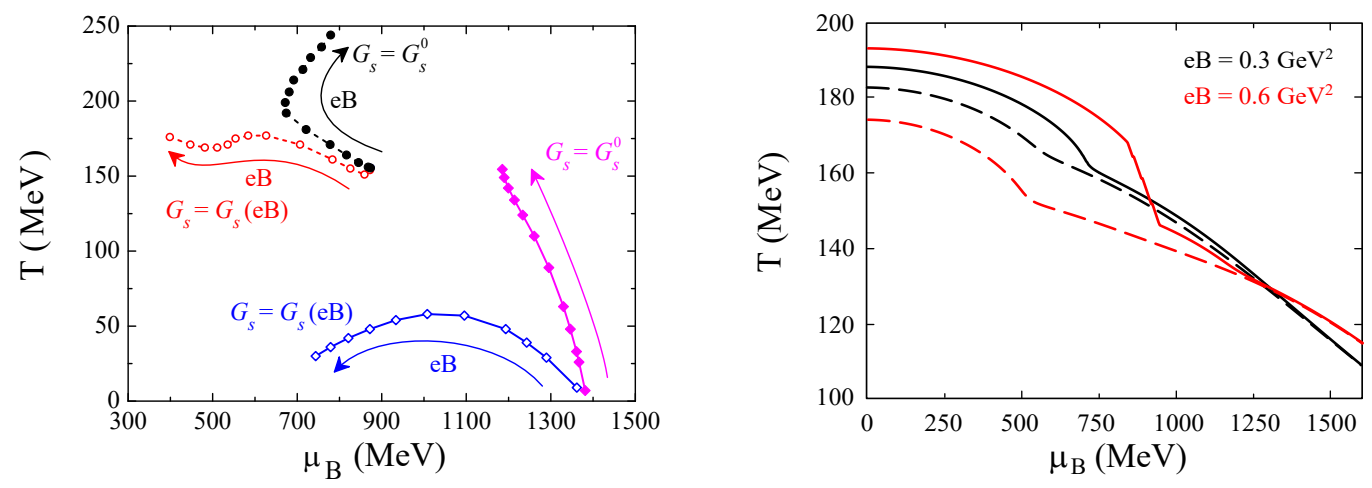

Figure 3: Left panel: CEPs of the light (red and black) and strange (blue and magenta) quarks as a function of $B$ for both scenarios: a constant coupling, $G_{s}^{0}$, and magnetic dependent coupling, $G_{s}(e B)$. The magnetic field increases from 0 to $1 \mathrm{GeV}^{2}$ in the arrows' directions. Right panel: $\Phi\left(T, \mu_{B}\right)=0.5$ when $G_{s}=G_{s}^{0}$ (full lines) and when $G_{s}(e B)$ (dashed lines) for $e B=0.3 \mathrm{GeV}^{2}$ (black lines) and $e B=0.6 \mathrm{GeV}^{2}$ (red lines).

Let us now focus our attention on the CEP of the strange sector. As we already saw, the presence of a magnetic field induces multiple CEPs. For both scenarios, we focus only on the CEP appearing at lower $\mu_{B}\left(\rho_{B}\right)$ in Fig. $\square$ that remains up to $e B \sim 1 \mathrm{GeV}^{2}$ (the CEP at higher $\mu_{B}$ disappears from the phase diagram at $e B \sim 0.4 \mathrm{GeV}^{2}$; similarly to the CEP for light sector [Q] ). The CEP's position shows a different behavior depending on the presence of an IMC mechanism: while at lower values of $B$ it moves towards lower $\mu_{B}$ in both scenarios, at high magnetic fields the $T^{C E P}$ increases monotonously with the intensity of the magnetic field for a constant coupling $G_{s}^{0}$, but $T^{C E P}$ is a decreasing function when we have $G_{s}(e B)$.

With increasing $B$, the position of the CEP in the scenario with $G_{s}(e B)$ (blue line) shows some similarity with the CEP of the light quarks (red line) by moving to lower $\mu_{B}$. For the constant coupling $G_{s}^{0}$ scenario (magenta line) the CEP goes to higher values of $T$ but lower values of $\mu_{B}$.

Finally, some considerations concerning the deconfinement transition. In the presence of a magnetic field the deconfinement transition is still a crossover, having an analytic behavior in opposition to a first-order phase transitions. The crossover transition thus allows for different def- 
initions of the pseudo-critical temperature. In the right panel of Fig. [1, we present the $\left(T, \mu_{B}\right)$ values where $\Phi\left(T, \mu_{B}\right)=0.5$, which is a possible way of defining a pseudo-critical temperature for deconfinement, with $G_{s}=G_{s}^{0}$ (full lines) and $G_{s}(e B)$ (dashed lines) for two magnetic field strengths: $e B=0.3 \mathrm{GeV}^{2}$ (black lines) and $e B=0.6 \mathrm{GeV}^{2}$ (red lines). We notice that the locations of the deconfinement transition is quite insensitive to the presence of an external magnetic field for both models. Furthermore, the analytic nature of the transition is preserved throughout the phase diagram.

\section{Acknowledgments}

This work was supported by 'Fundação para a Ciência e Tecnologia', Portugal, under the project No. UID/FIS/04564/2016 and under the Grants No. SFRH/BPD/102273/2014 (P.C.), and under the project CENTRO-01-0145-FEDER-000014 (MF) through CENTRO2020 program. This work was partly supported by 'NewCompstar', COST Action MP1304.

\section{References}

[1] J. O. Andersen, W. R. Naylor and A. Tranberg, Rev. Mod. Phys. 88 (2016) 025001, [arXiv:1411.7176 [hep-ph]].

[2] M. Ferreira, P. Costa, D. P. Menezes, C. Providência and N. Scoccola, Phys. Rev. D 89 (2014) no.1, 016002, [arXiv:1305.4751 [hep-ph]].

[3] M. Ferreira, P. Costa, O. Lourenço, T. Frederico and C. Providência, Phys. Rev. D 89 (2014) no.11, 116011, [arXiv:1404.5577 [hep-ph]].

[4] M. Ferreira, P. Costa and C. Providência, Phys. Rev. D 89 (2014) no.3, 036006, [arXiv:1312.6733 [hep-ph]].

[5] M. Ferreira, P. Costa and C. Providência, Phys. Rev. D 90 (2014) no.1, 016012, [arXiv:1406.3608 [hep-ph]].

[6] V. Skokov, A. Y. Illarionov and V. Toneev, Int. J. Mod. Phys. A 24 (2009) 5925, [arXiv:0907.1396 [nucl-th]].

[7] D. P. Menezes, M. B. Pinto, L. B. Castro, P. Costa and C. Providência, Phys. Rev. C 89 (2014) no.5, 055207, [arXiv:1403.2502 [nucl-th]].

[8] K. Enqvist and P. Olesen, Phys. Lett. B 319 (1993) 178, [hep-ph/9308270].

[9] P. Costa, M. Ferreira, H. Hansen, D. P. Menezes and C. Providência, Phys. Rev. D 89 (2014) no.5, 056013, [arXiv:1307.7894 [hep-ph]].

[10] P. Costa, M. Ferreira, D. P. Menezes, J. Moreira and C. Providência, Phys. Rev. D 92 (2015) no.3, 036012, [arXiv:1508.07870 [hep-ph]].

[11] P. Costa, Phys. Rev. D 93 (2016) no.11, 114035, [arXiv:1610.06433 [nucl-th]].

[12] S. P. Klevansky and R. H. Lemmer, Phys. Rev. D 39 (1989) 3478.

[13] G. S. Bali, F. Bruckmann, G. Endrodi, Z. Fodor, S. D. Katz, S. Krieg, A. Schafer and K. K. Szabo, JHEP 1202 (2012) 044, [arXiv:1111.4956 [hep-lat]].

[14] F. Bruckmann, G. Endrodi and T. G. Kovacs, JHEP 1304 (2013) 112, [arXiv:1303.3972 [hep-lat]].

[15] M. Ferreira, P. Costa and C. Providência, submitted for publication. 